\title{
Three-dimensionality and discriminability in the object-superiority effect
}

\author{
JAMES T. ENNS \\ University of British Columbia, Vancouver, British Columbia, Canada \\ and \\ AARON B. GLANI \\ Dalhousie University, Halifax, Nova Scotia, Canada
}

\begin{abstract}
Briefly presented target lines are identified more accurately in some contexts of additional noninformative lines than in other contexts (the object-superiority and object-line effects). One explanation for these effects is that the object-like appearance of some contexts (i.e., their threedimensionality and structural coherence) confers an advantage on the perceptual processing of individual line segments. An alternative explanation is that object-like contexts are simply more discriminable from one another than are other contexts. In the present study, subjects participated in three tasks involving the same set of contexts: tachistoscopic line-identification accuracy (Experiment 1), subjective ratings of three-dimensionality and dissimilarity under a range of exposure conditions (Experiments 1 and 2), and tachistoscopic same-different accuracy (Experiment 3). The main finding was that three-dimensionality and discriminability made equal and independent contributions to line-identification accuracy. Two secondary findings were: (1) that subjective dissimilarity ratings were unreliable indicators of tachistoscopic discriminability, and (2) that line-identification and same-different performance both supported a noisy-operator model of perceptual representation.
\end{abstract}

The object-superiority effect (OSE) refers to the wellknown finding that briefly presented target lines that differ in location and/or orientation are identified more accurately when embedded in some arrangements of context lines than when embedded in others. A closely related finding, the object-line effect (OLE), refers to a special case of the OSE in which target lines are identified more accurately in the context of other lines than when they are presented alone in the visual field. The present study examined the contributions of two stimulus properties to these effects: (1) the perceived three-dimensionality of the contexts, and (2) the perceptual discriminability of contexts that differ only in the location of the target line.

The OSE is of theoretical interest because the embedding context lines are identical for all target lines and thus cannot contribute any unique information about the target's identity. This suggests that the OSE is an example of the top-down influence that higher order stimulus properties can have on the perception of component parts (Navon, 1977; Palmer, 1977). The OLE is provocative in an additional way because it flies in the face of several

This research was funded by a grant from the Natural Sciences and Engineering Research Council of Canada. Portions of the data were presented as a MS thesis to Dalhousie University by A. B. Gilani. The authors are grateful to George Weir and Scott Lewin for running the subjects in Experiments $1 \mathrm{~A}$ and $1 \mathrm{C}$, and to several anonymous reviewers for critical suggestions on earlier drafts. Reprint requests may be addressed to J. T. Enns, Department of Psychology, University of British Columbia, 2136 West Mall, Vancouver, British Columbia V6T 1Y7, Canada. widely held assumptions concerning the effects of extraneous information on target identification. These include lateral masking by adjacent contours (Flom, Weymouth, \& Kahneman, 1963), a decrease in the signal-to-noise ratio (Estes, 1972; Estes \& Taylor, 1966), and the camouflaging effects of higher order stimulus structure (e.g., good form) on stimulus elements (Banks \& Prinzmetal, 1976; Gottschaldt, 1926; Millspaugh, 1978).

Most of the studies subsequent to the original demonstrations of the OSE (Weisstein \& Harris, 1974) and the OLE (Williams \& Weisstein, 1978) have been aimed at specifying the stimulus properties that may facilitate these effects. Proposals have included a number of properties that tend to be associated with meaningful objects: threedimensionality and coherence (Lanze, Maguire, \& Weisstein, 1985; Lanze, Weisstein, \& Harris, 1982; Weisstein, Williams, \& Harris, 1982), connectedness of contour (Chen, 1982), and structural relevance of target lines to the object (McClelland \& Miller, 1979). Other proposals have examined properties not specific to objects: haphazard poststimulus line masks (McClelland, 1978), and line detail at the point of fixation (Earhard, 1980; Earhard \& Armitage, 1980). In each proposal, the claim is that these properties affect the nature of the processing that a stimulus receives.

Although there have been convincing demonstrations that each of the foregoing properties has an effect on lineidentification accuracy, none of the properties have been shown to be either necessary or sufficient to produce a line-identification advantage. The OSE and OLE effects 
have been found with two-dimensional incoherent contexts (Enns \& Prinzmetal, 1984; McClelland \& Miller, 1979; Pomerantz, Sager, \& Stoever, 1977), with disconnected context lines (Earhard, 1980; Enns \& Prinzmetal, 1984), with structurally irrelevant target lines (Lanze et al., 1985; Lanze et al., 1982), without using a line mask (Klein, 1978; Williams \& Weisstein, 1978), and with line detail at the point of fixation (Weisstein et al., 1982).

As an alternative to the view that certain stimulus properties affect the type of processing received by individual lines, Enns and Prinzmetal (1984) presented an account of the OSE that did not make any claims for the processing priority of specific stimulus properties. Rather, these effects were said to be a function of two well-known factors in perceptual tasks: (1) the differential discriminability of contexts as the result of emergent perceptual features' being formed when target and context lines interact, and (2) the presence of a correlation between the emergent properties and the target lines themselves (i.e., feature redundancy).

The first claim is an acknowledgment that visual features other than individual line segments mediate perception in OSE experiments (Pomerantz et al., 1977). In particular, the interactions between context and target lines create such salient emergent features as line junctions, bounded regions, and three-dimensional corners that subjects are able to use to discriminate the target alternatives. The second claim reflects the idea that subjects must be able to use the emergent features diagnostically to identify the target lines.

To summarize this view, Enns and Prinzmetal (1984) made two specific predictions for the OSE. First, they hypothesized that line-identification accuracy would covary with the perceived dissimilarity between the contexts. Second, they proposed that there must be a consistent mapping between the target lines and the emergent features in a context for the line-identification advantage to emerge.

Enns and Prinzmetal (1984) provided evidence for this account in three experiments. Their first experiment used 10 different three-dimensional contexts to demonstrate that the OLE was critically dependent on the characteristics of the entire set of contexts that were presented to subjects. In the first phase of the experiment, a robust OLE was obtained for each of the contexts presented to subjects in blocked trials. In the second phase, the contexts were presented in mixed blocks, and accuracy was actually greater for the target lines alone than for target lines in the contexts.

Their second experiment systematically varied the degree of consistency between salient emergent features in two-dimensional lines-in-contexts (triangle vs. arrows) and the target lines. Identification accuracy was predicted by this consistency, thus supporting the second claim of Enns and Prinzmetal's account.
Their third experiment tested the claim that lineidentification accuracy was a function of the dissimilarity between the contexts. Two-dimensional contexts were rated for dissimilarity by one group of subjects, and these ratings reliably predicted the magnitude of the OLE for another group of subjects. Furthermore, the dissimilarity ratings predicted target accuracy only when the contexts were presented in blocked trials (i.e., when there was consistency between the emergent features and target lines).

In a recent study, Lanze et al. (1985) provided additional support for the claim that the OSE varies with context dissimilarity. Contexts were constructed that varied orthogonally in the presence/absence of emergent features (triangles, arrows) and in perceived dimensionality (two, three). The emergent features were consistently mapped to the two target lines, thus satisfying the second condition of Enns and Prinzmetal's (1984) proposal. The main finding was that contexts with salient emergent features yielded higher accuracy than those without these features. However, three-dimensional contexts also yielded higher accuracy than two-dimensional contexts. Lanze et al. (1985) concluded that three-dimensionality and dissimilarity contributed independently to the OSE.

Another recent study (Earhard, 1987) supported Enns and Prinzmetal's (1984) claim that the OSE varies with the degree of specification between salient emergent features and the target lines. Contexts were constructed such that salient emergent features either fully specified the target lines (correlation $=1.0$ ) or did not specify the target lines at all (correlation $=0$ ). Perceived dimensionality was also varied. A reliable contribution was found for both the degree of specification and three-dimensionality.

A potential problem with Lanze et al.'s (1985) and Earhard's (1987) conclusions is that in both studies there were no measures of context dissimilarity that were independent of the main dependent measure of lineidentification accuracy. This leaves open the possibility that the three-dimensional contexts were more discriminable than the two-dimensional contexts prior to the manipulations of the presence/absence of emergent features and target specification by emergent features. Without an independent measure of discriminability, it is not clear whether the results of either study showed an influence of three-dimensionality. Enns and Prinzmetal's (1984) experiments are also not helpful on this question, since in their studies target specification and dissimilarity were varied systematically only with two-dimensional contexts (Experiment 3).

The present study was designed to explore this question in addition to three more minor aspects of Enns and Prinzmetal's (1984) proposal. The first question we addressed concerned a direct comparison of perceived dimensionality (three vs. two) with dissimilarity (high vs. low). In Experiment 1, contexts were designed that varied orthogonally in these two factors, as measured by the sub- 
jects' ratings under free-viewing conditions. The contexts were then presented to other subjects in the standard lineidentification task used in OLE and OSE experiments.

The second question concerned a procedural difference between Enns and Prinzmetal's (1984) study and other studies of the OLE and OSE (e.g., Lanze et al., 1985; Lanze et al., 1982; Weisstein \& Harris, 1974; Williams \& Weisstein, 1978). In most studies, the target lines were presented in known locations in the display and very close to the fixation point (less than $0.5^{\circ}$ of arc). However, in Enns and Prinzmetal's (1984) second and third experiments, the target lines were presented in one of four locations, $1.25^{\circ}$ of arc from fixation, on each trial. It is possible that, since the fovea and the parafovea are sensitive to different stimulus properties (Ambler \& Finklea, 1976; Beck, 1972), the Enns and Prinzmetal results do not generalize to targets present at the fovea. This question was also addressed in Experiment 1 by comparing the OLE and the OSE for target lines presented at the fovea and for the same lines presented with locational uncertainty in the parafovea.

Third, this study examined subjects' ratings of stimulus properties under several different exposure conditions. Past studies of the effects of three-dimensionality (e.g., Lanze et al., 1985; Lanze et al., 1982; Weisstein et al., 1982) and dissimilarity (Enns \& Prinzmetal, 1984) have measured these properties with ratings made under freeviewing conditions. However, dissimilarity ratings of visual patterns made under free-viewing conditions do not always generalize to ratings made under brief exposure conditions (Klein, 1982). Thus, it is important to know whether generality is also lacking for the ratings used in OSE experiments. This question was addressed in Experiment 2 by comparing dissimilarity and dimensionality ratings in a free-viewing condition with two brief exposure conditions (100) and $40 \mathrm{msec}$ ).

The fourth question concerned the relations between the OSE, ratings of stimulus properties, and a performance measure of discriminability. Enns and Prinzmetal (1984) used dissimilarity ratings to estimate the perceptual discriminability of their contexts under the assumption that these two constructs were highly related (e.g., Beck, 1982). This assumption, however, has not been tested directly for the stimuli used in OSE experiments; therefore, we compared a same-different discrimination task in Experiment 3 with the subjective ratings of dissimilarity in Experiment 2.

\section{EXPERIMENT 1}

In Experiment 1, we examined the relative contributions of perceived three-dimensionality and dissimilarity on the OLE and OSE. Following previous studies (Enns \& Prinzmetal, 1984; Lanze et al., 1985; Lanze et al., 1982; Weisstein et al., 1982), subjective ratings of both factors under free-viewing conditions were first obtained from one group of subjects; a second group of subjects then participated in a tachistoscopic line-identification task.

The line-identification task was divided into three subexperiments, each with a different sample of subjects (Experiments $1 \mathrm{~A}, 1 \mathrm{~B}, 1 \mathrm{C})$. It was necessary to subdivide the experiment because the full experiment would have required $9 \mathrm{~h}$ of participation per subject, and the Dalhousie University subject pool restricted student participation to a total of $3 \mathrm{~h}$. One pair of subexperiments (1A and 1B) examined the effect of locational uncertainty of the target. Another pair of subexperiments compared the generality of the results for eight contexts to a second set of eight contexts (1A vs. 1C).

\section{General Method}

Rating task. The 16 contexts shown in Figure 1 were designed to vary orthogonally in perceived three-dimensionality and dissimilarity. Stimuli were formed by combining the two target lines, $A$ and $B$, with the different contexts. Each context was drawn in black ink in the center of a $13 \times 7.5 \mathrm{~cm}$ white index card. The target lines were $0.7 \mathrm{~cm}$ in length, the total line length of the context lines ranged from 6.0 to $7.0 \mathrm{~cm}$, and the horizontal distance between the two target lines on each card was $2.9 \mathrm{~cm}$.

Five of 10 raters received dimensionality-rating instructions first, followed by dissimilarity-rating instructions. The other 5 raters received the instructions in the reverse order. In the dimensionalityrating task, the subjects were instructed to look through a shuffled deck of 16 cards, noting that some figures appeared more threedimensional than others. They were then asked to assign a value of 10 to the best example of a three-dimensional figure, and a value of 0 to the figure that appeared most flat, or two-dimensional. These two cards were set aside, and the rater proceeded through the reshuffled deck of cards, assigning values of 0 through 10 to each figure

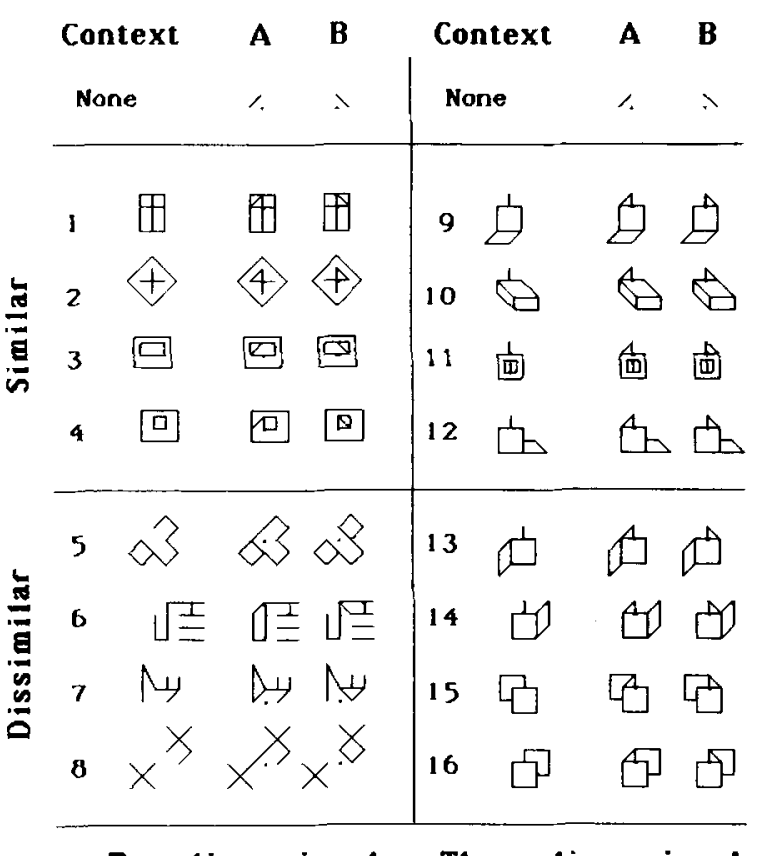

Two-dimensional

Three-dimensional

Figure 1. The two target lines and 16 contexts used in this study. 
to indicate their perceived three-dimensionality. In the dissimilarityrating task, the subjects were asked to rate the same figures for dissimilarity. This time, the raters were asked to look through the reshuffled deck of cards, noting that some pairs of figures appeared more dissimilas/similar to one another. A value of 10 was assigned to the most dissimilar pair and a value of 0 was assigned to the most similar pair. The rater then assigned values of 0 through 10 to the remaining cards in the deck to indicate their perceived dissimilarity.

The mean dimensionality and dissimilarity ratings for each of the 16 contexts are shown as a scattergraph in Figure 2. Vertical bars represent the standard errors for the mean dissimilarity ratings; horizontal bars represent the standard errors for the mean threedimensionality ratings. As shown in the graph, the raters confirmed the intuitions we had used to design these contexts. First, the contexts were judged to vary in their perceived three-dimensionality and dissimilarity in a consistent fashion: the mean ratings for the 16 contexts showed reliable differences for both rated threedimensionality $[F(15,135)=34.57, p<.01, M S e=3.525]$ and rated dissimilarity $[F(15,135)=18.25, p<.001, M S e=4.340]$. Second, the variation in these ratings was mutually orthogonal $[r(14)$ $=0.07, p>.20]$, which indicates that these two perceptual attributes were not correlated in the present set of contexts.

Line-identification task. The displays used for the tachistoscopic line-identification task included each member of the 16 contexts shown in Figure 1, as well as the individual target lines A and B. Each contexi was drawn in black ink on a standard white index card and was presented in one field of a three-field Gerbrands tachistoscope (Model T3B1). The target lines subtended $0.5^{\circ}$ of arc. Each target presentation was preceded and followed by a haphazard line mask, similar in construction to those used in previous experiments (Enns \& Prinzmetal, 1984; McClelland, 1978). There were a total of 350 lines in the mask, which filled the display area seen by the subjects $\left(9^{\circ} \times 9^{\circ}\right.$ of arc). The mask was presented together with the fixation point in the second field of the tachistoscope. The stimulus and mask fields were equated for luminance at $110 \mathrm{~cd} / \mathrm{m}^{2}$ (range over days was $100-120 \mathrm{~cd} / \mathrm{m}^{2}$ ).

Each trial began with the masking field illuminated. The experimenter initiated the trial by saying "ready" and then pressing a switch that began the trial sequence. Following a 500 -msec delay, the stimulus field was illuminated for $40 \mathrm{msec}$, and then the masking field was illuminated again. The subject responded verbally " $A$ " or " $B$ " in response to the target line in each context.

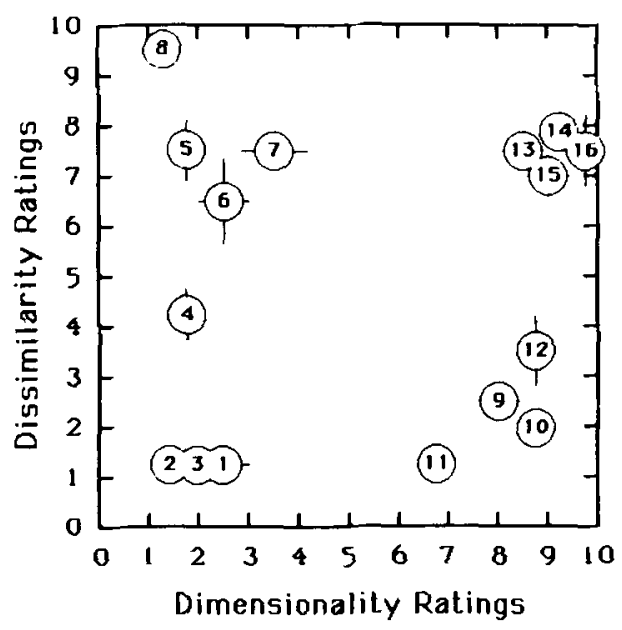

Figure 2. Scattergraph of the relation between dimensionality and dissimilarity ratings in Experiment 1.
The subject was instructed to respond as accurately as possible and to guess when uncertain. Reaction time was not recorded.

In each experiment, the observers participated in three 1-h sessions on separate days in order to receive target line presentations in all nine contexts. Each context was represented by 120 trials for a total of 1,080 trials for each subject. Each context was introduced with a review of the figures to be presented. This was followed by 24 practice trials in which the stimulus-exposure duration was gradually reduced from 100 to $40 \mathrm{msec}$. The 96 test trials were then run in four blocks of 24 trials with the exposure duration set at $40 \mathrm{msec}$. The trials within each block were evenly divided between target lines $\mathbf{A}$ and $\mathbf{B}$, and random presentation was ensured by thoroughly shuffling the stimulus cards before each block of trials. Feedback was provided at the end of each block and was available within a block at the request of the subject. The order of contexts was counterbalanced across subjects using a complete Latin square design.

Subjects. Ten undergraduates at Dalhousie University participated in the rating task. A separate group of 36 undergraduates participated in the line-identification task, with 12 subjects assigned to each of Experiments 1A, 1B, and 1C. All subjects were naive as to the purpose of the experiment and received partial course credit for participation.

\section{Experiment 1A}

Experiment 1A examined line-identification accuracy among eight contexts that the ratings suggested were the best exemplars of the factors of interest: two-dimensional similar (Contexts 1 and 2), two-dimensional dissimilar (Contexts 5 and 6), three-dimensional similar (Contexts 9 and 10), and three-dimensional dissimilar (Contexts 13 and 14). Two criteria were used to select these initial eight contexts: (1) Contexts representing different experimental factors should be maximally different from one another, and (2) the subjective ratings of the two stimulus properties should be kept as orthogonal as possible. The target lines alone were included as a ninth context. The target lines in all contexts were centered around the fixation point, as shown in Figure 1.

Results. The first column of data in Table 1 shows the proportion of correct line identifications for the nine contexts in Experiment 1A. In the table, we indicate the contexts that yielded an OLE (context accuracy minus singleline accuracy) reliably larger than zero. All of the threedimensional contexts yjelded an OLE, whereas only one of the two-dimensional contexts did so. A repeated measures analysis of variance (ANOVA) examined the OSE (context accuracy), with dimensionality (three, two) and dissimilarity (dissimilar, similar) as the factors of interest. The only significant result was that accuracy was greater for three-dimensional contexts than for two-dimensional contexts $[F(1,11)=28.43, p<.001, M S e=.01089]$. The dissimilarity effect and the dimensionality $x$ dissimilarity interaction yielded $F$ values of $<1.0, M S e=$ .01266 , and $2.29, M S e=.00954$, respectively.

\section{Experiment 1B}

Experiment 1B was a replication of Experiment $1 \mathrm{~A}$ with the exception that target lines appeared randomly at one of four locations around the fixation point. The center of each target line was $1.25^{\circ}$ of arc from fixation. A com- 
Table 1

Line-Identification Accuracy in Experiment 1 (Proportion Correct)

\begin{tabular}{|c|c|c|c|c|c|c|c|}
\hline \multirow[b]{2}{*}{ Context } & \multicolumn{2}{|c|}{ Experiment $1 \mathrm{~A}$} & \multicolumn{2}{|c|}{ Experiment 1B } & \multicolumn{2}{|c|}{ Experiment $1 \mathrm{C}$} & \multirow[b]{2}{*}{$z$ score } \\
\hline & $M$ & $S D$ & $M$ & $S D$ & $M$ & $S D$ & \\
\hline \multicolumn{8}{|c|}{ Two-Dimensional/Similar } \\
\hline 1 & .585 & .09 & .747 & .12 & & & -1.577 \\
\hline 2 & .668 & .13 & $.878^{*}$ & .13 & & & -.408 \\
\hline 3 & & & & & 669 & .14 & -1.194 \\
\hline 4 & & & & & 680 & .13 & -1.016 \\
\hline \multicolumn{8}{|c|}{ Two-Dimensional/Dissimilar } \\
\hline 5 & $.675^{*}$ & .17 & $.844 *$ & .14 & & & -.310 \\
\hline 6 & .633 & .07 & $.828 *$ & .11 & & & -.901 \\
\hline 7 & & & & & .690 & .16 & -.855 \\
\hline 8 & & & & & $.713 \dagger$ & .21 & -.484 \\
\hline \multicolumn{8}{|c|}{ Three-Dimensional/Similar } \\
\hline 9 & $.751^{*}$ & .13 & $.949 *$ & .04 & & & .761 \\
\hline 10 & $.790^{*}$ & .14 & $.889 *$ & .12 & & & 1.310 \\
\hline 11 & & & & & $.833 *$ & .09 & 1.452 \\
\hline 12 & & & & & $.792^{*}$ & .15 & .790 \\
\hline \multicolumn{8}{|c|}{ Three-Dimensional/Dissimilar } \\
\hline 13 & $.699^{*}$ & .12 & $.891^{*}$ & .16 & & & .028 \\
\hline 14 & $.775^{*}$ & .16 & $.852 *$ & .13 & & & 1.099 \\
\hline 15 & & & & & $.781^{*}$ & .14 & .613 \\
\hline 16 & & & & & $.788^{*}$ & .15 & .726 \\
\hline \multicolumn{8}{|c|}{ Single Target Lines } \\
\hline & .535 & .08 & .726 & .13 & .637 & .12 & \\
\hline
\end{tabular}

Note-Significant OLE (difference in accuracy between contexts and single target lines): ${ }^{*} p<.01$. $\dagger p<.05$.

parison of these results with Experiment $1 \mathrm{~A}$ would indicate whether the factors of locational uncertainty and/or foveal eccentricity played a role in the OSE.

Results. Table 1 shows the proportion of correct line identifications for the nine contexts in Experiment 1B. All three-dimensional contexts and three-quarters of the twodimensional contexts yielded a reliable OLE. The ANOVA for the OSE revealed that accuracy was reliably greater for three-dimensional contexts than for twodimensional contexts $[F(1,11)=9.81, p<.01, M S \mathrm{e}=$ $.01237]$, and that accuracy did not differ between dissimilar and similar contexts $[F(1,11)<1.0, M S e=.00442]$. However, there was a marginal dimensionality $\times$ dissimilarity interaction $[F(1,11)=4.01, p<.08, M S e=$ $.00771]$, indicating that for three-dimensional contexts, similar contexts ( 9 and 10 ) had slightly higher accuracy rates than did dissimilar contexts (13 and 14), whereas for two-dimensional contexts, dissimilar contexts (5 and 6) had slightly higher accuracy rates than did similar ones (1 and 2).

An ANOVA comparing the results of Experiments 1A and $1 B$ revealed three reliable effects. Accuracy was reliably greater overall in Experiment $1 \mathrm{~B}$ than in Experiment $1 \mathrm{~A}[F(1,22)=18.20, p<.001, M S e=.06983]$; three-dimensional contexts yielded reliably greater accuracy than did two-dimensional contexts $[F(1,22)=$ $35.20, p<.001, M S e=.01163]$, and the dimensionality $\times$ dissimilarity interaction was reliable $[F(1,22)=$ $6.07, p<.03, M S e=.00862]$. This interaction reflected a small but consistent trend for dissimilar contexts to yield lower accuracy than similar contexts when they were three-dimensional (mean difference $=4.1 \%$ ) and greater accuracy when the contexts were two-dimensional (mean difference $=2.6 \%$ ). No other effects were close to being reliable $(p>.20)$. A comparison of the accuracy in the no-context condition indicated that single-line accuracy was also reliably greater in Experiment $1 \mathrm{~B}$ than in Experiment $1 \mathrm{~A}[F(1,22)=17.37, p<.01, M S \mathrm{e}=$ $.01260]$.

\section{Experiment 1C}

Experiment $1 \mathrm{C}$ examined the eight contexts that had not been tested in Experiment 1A, in an effort to increase the generality of the results. An inspection of Figure 2 showed that these contexts could also be grouped, albeit somewhat less orthogonally, according to the factors of interest: two-dimensional similar (Contexts 3 and 4), twodimensional dissimilar (Contexts 7 and 8), threedimensional similar (Contexts 11 and 12), and threedimensional dissimilar (Contexts 15 and 16). As in Experiment $1 \mathrm{~A}$, the single target lines were included as the ninth context and the target lines in all contexts were presented at a single location centered on the fixation point.

Results. Table 1 shows the proportion of correct line identifications for the nine contexts in Experiment 1C. A reliable OLE was found for each three-dimensional context and for one-fourth of the two-dimensional contexts as indicated. An ANOVA of the OSE revealed that accuracy was reliably greater for three-dimensional contexts 
than for two-dimensional contexts $[F(1,11)=24.53$, $p<.001, M S e=.01195]$. The dissimilarity effect and the dimensionality $\times$ dissimilarity interaction were both not reliable $[F(1,11)<1.0, M S e=.01025$, and $F(1,11)$ $=1.06, M S e=.01718$, respectively $]$.

An ANOVA comparing the results of Experiments $1 \mathrm{~A}$ and $1 C$ revealed only one reliable effect: threedimensional contexts yielded reliably greater accuracy than did two-dimensional contexts $[F(1,22)=52.78$, $p<.001, M S \mathrm{e}=.01142]$. The dimensionality $\times$ dissimilarity interaction was marginally reliable $[F(1,22)=$ $2.99, p<.03, M S e=.01336]$, reflecting a trend for dissimilar contexts to yield lower accuracy than similar contexts when they were three-dimensional (mean difference $=3.1 \%$ ) and greater accuracy when the contexts were two-dimensional (mean difference $=2.7 \%$ ). The $F$ ratios of all other effects were $<1.0$. A comparison of the accuracy for single target lines indicated that accuracy was somewhat greater in Experiment 1C than in Experiment $1 \mathrm{~A}[F(1,22)=4.89, p<.05, \mathrm{MSe}=.01190]$.

\section{Discussion}

A consistent result in Experiment 1 was that the rated three-dimensionality of the contexts reliably predicted the OSE and OLE. This finding is consistent with many previous experiments on the role of three-dimensionality in the OSE and OLE (Lanze et al., 1985; Lanze et al., 1982; Weisstein et al., 1982). A unique finding of the present study was that this result held even when the rated dissimilarity of the contexts was controlled (cf. Earhard, 1987; Lanze et al., 1985). These findings support Weisstein's claim that three-dimensional contexts are at a processing advantage relative to two-dimensional contexts (Lanze et al., 1985; Lanze et al., 1982; Weisstein et al., 1982). On the other hand, there was no evidence to support Enns and Prinzmetal's (1984) claim that rated dissimilarity predicted the OSE or the OLE when dimensionality was controlled.

An interaction between dimensionality and dissimilarity was evident in Experiment 1B and in the combined analyses for Experiments $1 \mathrm{~A}$ and $1 \mathrm{C}$. These effects reflected a small but consistent trend for dissimilarity to be positively predictive of the OSE for two-dimensional contexts and negatively predictive for three-dimensional contexts. Although the trend for the two-dimensional contexts was consistent with the results of Enns and Prinzmetal (1984), the finding of a trend in the opposite direction for the three-dimensional contexts was clearly counter to the claim that line identification could be predicted by rated context dissimilarity.

There was no evidence in Experiment 1 that locational uncertainty and/or the eccentricity of the target line affected the pattern of OSE and OLE results. However, accuracy was reliably greater overall when the target lines were presented at four possible locations in the parafovea (Experiment 1B) than when they were presented at a sin- gle location at the fovea (Experiment 1A). This difference in overall accuracy may have reflected one or more of the differences between the two subexperiments. For instance, the two groups of subjects may have differed in their baseline visual acuity, the target lines may have been masked less heavily in the parafoveal locations than at the fovea, or the foveal location may have contained line detail close enough to the fixation point to adversely affect line processing (Earhard, 1980). In any event, the important finding was that the procedural difference between Enns and Prinzmetal's (1984) study and other studies of the OSE did not bear, in an important way, on the relative accuracy levels for various lines-in-context.

The similarity of the results in Experiments $1 \mathrm{~A}$ and $1 \mathrm{C}$ further indicated that these findings were not unique to a particular set of eight contexts. The generality of the pattern of data in Experiment $1 \mathrm{~A}$ to a new set of contexts in Experiment $1 \mathrm{C}$ gave us confidence that subjective ratings of three-dimensionality predicted line-identification accuracy whereas subjective ratings of dissimilarity did not.

\section{EXPERIMENT 2}

Experiment 2 explored the possibility that the predictive power of three-dimensionality and dissimilarity ratings varied with the exposure conditions under which they were made. For example, it is possible that the stimulus attributes responsible for perceptual dissimilarity under brief exposure conditions become salient only when the contexts are also rated under brief viewing conditions (see Klein, 1982).

\section{Method}

Procedure. In this experiment, the 16 contexts in Figure 1 were rated for perceived three-dimensionality and dissimilarity under three exposure conditions: free viewing, $100 \mathrm{msec}$, and $40 \mathrm{msec}$. Each context pair was presented in the tachistoscope, using the lighting conditions, stimulus dimensions, line mask, and procedure of the previous line-identification experiments. The displays were drawn such that one member of a context pair lay on each side of the fixation point, with the center of the target lines $1^{\circ}$ of arc from fixation. Each pair was drawn twice, once with the $A$ member of the pair on the left and once with the A member on the right. Thus, there were 32 displays for the subjects to rate.

The subjects in each of the rating-instruction conditions were presented with all contexts in a random order and then asked to note that some pairs of figures appeared more similar/dissimilar to one another (or three-dimensional/two-dimensional) than did other pairs. The exposure duration for this preparatory phase was consistent with the condition to which each rater had been assigned: free viewing, $100 \mathrm{msec}$, and $40 \mathrm{msec}$, respectively. During the testing phase, the contexts were presented again in a new random order, and the subjects were instructed to assign values of 10 to the most dissimilar (or three-dimensional) context, values of $\mathbf{0}$ to the most similar (or two-dimensional) context, and intermediate values to contexts that fell between these extremes.

Subject. Ninety Dalhousie undergraduates received partial course credit for their participation as raters. Fifteen raters were assigned 

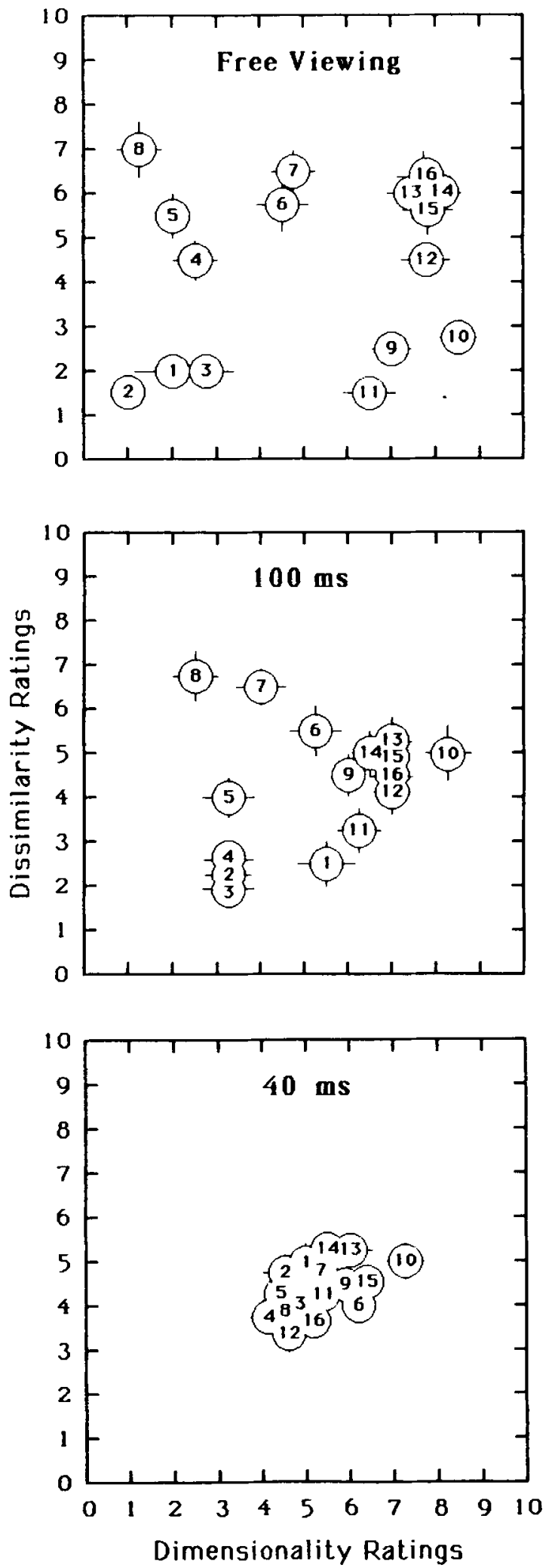

Figure 3. Scattergraphs of the relation between dimensionality and dissimilarity ratings as a function of viewing time in Experiment 2. to each of the six exposure-duration $\times$ rating-instruction conditions. Each rater participated in one session lasting approximately $15 \mathrm{~min}$.

\section{Results}

Stimulus property ratings. The mean ratings of dimensionality and dissimilarity in the three viewing conditions are shown as scattergraphs in Figure 3. Vertical bars represent the standard errors for the mean dissimilarity ratings; horizontal bars represent the standard errors for the mean three-dimensionality ratings.

Three main findings can be seen in the scattergraphs. First, the free-viewing ratings that were obtained in Experiment 1 with a repeated measures design were replicated here with a between-groups design. Contexts were judged to vary in perceived three-dimensionality and dissimilarity in a consistent fashion [dimensionality, $F(15,210)=32.62, p<.001, M S e=7.163$; dissimilarity, $F(15,210)=22.63, p<.001, M S e=5.426]$, and the variation in these ratings was mutually orthogonal $[r(14)=0.14, p>.20]$. Furthermore, the correlations between the free-viewing ratings in Experiment 1 and those in Experiment 2 were very high [dimensionality, $r(14)=.96, p<.01$; dissimilarity, $r(14)=.98$, $p<.01]$.

Second, the ratings made in the $100-\mathrm{msec}$ condition varied consistently across subjects [dimensionality, $F(15,210)=17.18, p<.001, M S e=6.200$; dissimilarity, $F(15,210)=12.02, p<.001, M S e=5.320]$ and were also orthogonal $[r(14)=0.18, p>.20]$, but the range of the dissimilarity ratings was substantially decreased relative to that in the free-viewing condition. This finding suggested that the subjects were less sensitive in this condition to the attributes on which the freeviewing dissimilarity judgments were based.

Finally, the ratings made in the $40-\mathrm{msec}$ condition showed an almost complete lack of consistency across subjects for both attributes [dimensionality, $F(15,210)=$ $1.58, M S e=5.383$; dissimilarity, $F(15,210)=1.13, M S e$ $=5.134]$. Under free-viewing conditions, even simple contrasts between the contexts judged to be threedimensional (dissimilar) and those judged to be twodimensional (similar) yielded only marginal evidence that the subjects were sensitive to dimensionality $[F(1,210)=$ $3.53, p<.10, M S e=5.383$, and no evidence of sensitivity to dissimilarity $[F(1,210)<1, M S e=5.134]$.

This third finding suggests that the subjects were unable to judge reliably the presence/absence of threedimensionality under brief exposure conditions, even though this property had a strong effect on lineidentification accuracy under the same exposure conditions in Experiment 1. One explanation for this result is that the subjects did not have sufficient familiarity with the contexts in Experiment 2. The subjects received four presentations of each of the 16 contexts in Experiment 2, for a total of 64 trials. In Experiment 1, each subject was presented with eight different contexts, for a total of 1,080 trials. 
To examine this explanation, the line-identification data from only the first block of trials in Experiment 1 were analyzed. In the first block of trials for each context in Experiment 1 , the subjects received 12 presentations of each member of the context, for a total of 192 trials. The results for these trials alone were unchanged from the overall analyses in Experiment 1 . In each subexperiment, lines were identified more accurately in three-dimensional than in two-dimensional contexts [Experiment 1 A, $F(1,11)=13.51, p<.01$; Experiment $1 \mathrm{~B}, F(1,11)=$ $14.37, p<.01$; Experiment $1 \mathrm{C}, F(1,11)=12.98$, $p<.01$, whereas the dissimilarity of the contexts had no effect on accuracy $(p>20)$.

Relations between ratings and line-identification accuracy. In order to examine the relations between line identification in Experiment 1 and the subjective ratings in Experiment 2 for the full set of 16 contexts, it was necessary to combine the accuracy data from Experiments IA and IC. However, the means of the accuracy data from the two subexperiments could not be combined directly because the overall accuracy was somewhat higher in Experiment $1 \mathrm{C}$ than in Experiment 1A (see results of Experiment $1 C$ ). To make the accuracy scores in the two subexperiments comparable, $z$ scores based on the mean and standard deviation in each subexperiment were used as the accuracy score for each context. This equated the two subexperiments for mean accuracy (mean $z=0$ ), but otherwise preserved the interval relations between contexts within each subexperiment. The $z$ scores of lineidentification accuracy for contexts 1-16 in Experiments $1 \mathrm{~A}$ and $1 \mathrm{C}$ are shown in Table 1 .

Table 2 shows the correlations between the subjective ratings of dimensionality and dissimilarity in Experiment 2 and the $z$ scores of line-identification data in Experiment 1 . In addition to the simple correlations, the partial correlations between each rated stimulus property (with the other rated stimulus property held constant) and line-identification accuracy are presented. The agreement between these two types of correlational measures is a reflection of the orthogonality of the two rated stimulus properties. As can be seen in Table 2, only the dimensionality ratings were reliable predictors of the OSE. Table 2 also shows that the reliability of these ratings

Table 2

Simple and Partial Correlations Between the Subjective Ratings in Experiment 2 and $z$ Scores of Line-Identification Accuracy in Experiment 1

\begin{tabular}{lccccccc}
\hline & \multicolumn{2}{c}{ Dimensionality Ratings } & & \multicolumn{3}{c}{ Dissimilarity Ratings } \\
\cline { 2 - 4 } \cline { 6 - 7 } Correlation & FV & 100 & 40 & & FV & 100 & 40 \\
\hline Simple & $.80^{*}$ & $.74 \dagger$ & .40 & -.03 & -.23 & .23 \\
Partial & $.82^{*}$ & $.72 \dagger$ & .38 & & -.14 & .09 & .05 \\
\hline
\end{tabular}

Note-Partial correlations are between ratings of one stimulus property (e.g., dimensionality) and line-identification accuracy, with the ratings of the other stimulus property (e.g., dissimilarity) controlled. FV = free viewing, $100=100$-msec exposure, $40=40$-msec exposure. Reliable correlation coefficients: ${ }^{*} r(14)=.62, p<.01 . \quad \operatorname{tr}(14)=.50$, $p<.05$. declined as the exposure duration under which the ratings were made was reduced.

\section{Discussion}

The main finding of Experiment 2 was that ratings of stimulus properties made under exposure conditions comparable to those in the line-identification task did not correlate with line-identification accuracy. Instead, the correlation between line-identification accuracy and dimensionality (the one stimulus property that correlated quite well under free-viewing rating conditions) decreased monotonically with decreasing exposure duration.

The main effect of decreased exposure on both dimensionality and dissimilarity ratings was a reduction in the range of values the subjects used to make their ratings. At the shortest exposure condition ( $40 \mathrm{msec}$ ), which was chosen to duplicate the conditions under which lines are identified in OSE experiments, the subjects were no longer able to discriminate reliably three-dimensional from twodimensional contexts, or dissimilar from similar contexts. A control analysis based on the data from only the first block of trials of line-identification data in Experiment 1 suggested that this result was not simply an artifact of the smaller number of context exposures that the subjects received in the rating tasks.

One implication of these findings is that the subjects in OSE experiments do not have introspective access to the stimulus properties that mediate line-identification accuracy, and thus, the OSE is not mediated by conscious decisions or strategies. Instead, it gives us confidence that the stimulus properties under investigation are relevant to the "unconscious inferences" of form perception (Helmholtz, 1867/1962).

\section{EXPERIMENT 3}

The discriminability of stimuli can be assessed in a number of ways. The assumption behind the use of subjective ratings in previous OSE studies (Enns \& Prinzmetal, 1984; Lanze et al., 1982; McClelland \& Miller, 1979; Weisstein et al., 1982) was that context dissimilarity ratings corresponded closely to context discriminability under brief exposure conditions. There is reason to suspect that this assumption does not always hold. For example, although ratings of subjective grouping and discrimination accuracy are highly related measures of texture segregation (Beck, 1982), dissimilarity ratings of individual forms do not always predict the ease with which those forms can be found in a multiform array (Beck, 1982; Julesz, 1981). Experiment 3 tested this assumption directly for the present contexts by comparing discrimination accuracy in a tachistoscopic same-different task with the dissimilarity ratings made in Experiment 2.

In addition to testing this important assumption, there are two other benefits of testing the OSE with a discrimination paradigm. The first is that it allows a test of the hypothesis that the three-dimensional contexts are more 
discriminable than two-dimensional contexts. Enns and Prinzmetal (1984) speculated that this was a possible source of the OLE, and, in the introduction, we speculated that this was a possible confound in both Earhard's (1987) and Lanze et al.'s (1985) studies. The present experiment permitted a direct test of this hypothesis.

The second benefit of the discrimination paradigm is that it permits a new look at the processes involved in the OSE. There are two models of perceptual matching that can be differentiated on the basis of the relative accuracy of same versus different trials in a discrimination task. The missing-feature model of perceptual matching proposes that a decision is made on the basis of both the shared and the distinctive features of two stimuli (Eriksen, O'Hara, \& Eriksen, 1982; Krueger \& Chignell, 1985). In this model, the early representations of a stimulus are relatively undifferentiated, or blob-like, and thus look similar. The detection of differences must await later analytic processing to fill in the missing features (Lockhead, 1972; Navon, 1977). This model predicts a tendency for false-same responses (lower accuracy on different than on same trials) in the early stages of processing because all percepts will begin with similar representations, no matter how different the stimuli.

The noisy-operator model claims that perceptual matches are based only on the number of feature differences that are detected in comparing two stimuli (Chignell \& Krueger, 1984; Krueger, 1978). The featuredifference counting process is inherently noisy because of the feature perturbations in the early representations of a stimulus (Prinzmetal, 1981; Treisman \& Schmidt, 1982; Wolford, 1975). The net result of this internal noise is to make it more likely that a same pair of stimuli will look different (only one perturbation is required) than that a different pair of stimuli will look the same (as many perturbations are required as there are differences). Thus, the main prediction of the noisy-operator model is a tendency toward false-different responses under conditions of uncertainty (i.e., lower accuracy on same than on different trials).

If the missing-feature model of perceptual matching were to fit the discrimination data best, it would be consistent with top-down explanations of the OSE (Pomerantz et al., 1977; Weisstein \& Harris, 1974). That is, the line-identification advantage is the result of more rapid processing of certain higher order stimulus properties (e.g., three-dimensional surfaces and comers, good Gestalt forms) than of other properties (e.g., twodimensional line relations, poor Gestalt forms), thereby allowing lower order features (e.g., line segments) to be represented more rapidly.

Alternatively, if the noisy-operator model were to fit the data best, it would be consistent with Enns and Prinzmetal's (1984) proposal. This view of the OSE does not make any claims for the special processing status of certain stimulus properties. It asserts only that emergent features act as additional sources of information that observers can use to assist in the identification of target lines.
The more feature differences, emergent or otherwise, that exist between contexts, the easier it will be for observers to diagnose the correct target line.

\section{Method}

Stimuli. The 16 contexts shown in Figure 1 were used to create 64 different displays in this experiment. Each display consisted of a context pair with one member on each side of the fixation point. The target line in each pair member was $0.5^{\circ}$ of arc in length and $1.0^{\circ}$ of arc from fixation. Sixteen displays contained member $A$ on the left of fixation and member $B$ on the right, 16 displays contained B on the left and A on the right, 16 displays contained A on both sides of fixation, and 16 displays contained $B$ on both sides of fixation. Thus, half of the displays contained the same target and half contained different targets on each side of fixation. The line mask used in Experiments 1 and 2 again appeared before and after display presentations.

Procedure. Each subject received 384 trials in six blocks of 64 trials. Within a block, each display was presented once in random order. The subject's task on each trial was to indicate verbally whether the target lines on either side of fixation were "the same" or "different." Following each block of trials, accuracy was recorded and the exposure duration was adjusted between 40 and $60 \mathrm{msec}$ in 5-msec steps to maintain an overall accuracy level of $70 \%-80 \%$ correct. Feedback was provided at the end of each block and was available within a block at the request of the subject. Each session began with a review of the displays to be presented and a block of 64 practice trials. During this practice block, the exposure duration was gradually decreased from 200 to $50 \mathrm{msec}$.

Subjects. Twelve Dalhousie undergraduates, naive as to the purpose of the experiment, received partial course credit for participating in a single $1-\mathrm{h}$ session in this experiment.

\section{Results}

The mean proportion accuracy for each of the contexts is shown in Table 3. A repeated measures ANOVA examined the factors of context (1-16) and trial type (same, different). There were reliable differences in accuracy across the 16 contexts $[F(15,165)=18.92, p<.001$, $M S e=.01928]$; same trials yielded reliably lower accuracy than did different trials $[F(1,11)=32.79$,

Table 3

Same-Different Accuracy in Experiment 3 (Proportion Correct)

\begin{tabular}{|c|c|c|c|c|c|c|}
\hline \multirow[b]{2}{*}{ Context } & \multicolumn{2}{|c|}{ Same } & \multicolumn{2}{|c|}{ Different } & \multicolumn{2}{|c|}{ Combined } \\
\hline & $M$ & $S D$ & $M$ & $S D$ & $M$ & $S D$ \\
\hline 1 & .797 & .18 & .631 & .22 & .714 & .20 \\
\hline 2 & .791 & .17 & .833 & .08 & .812 & .15 \\
\hline 3 & .614 & .18 & .794 & .13 & .704 & .16 \\
\hline 4 & .645 & .15 & .867 & .11 & .756 & .10 \\
\hline 5 & .540 & .16 & .804 & .20 & .672 & .17 \\
\hline 6 & .665 & .14 & .401 & .17 & .533 & .15 \\
\hline 7 & .507 & .17 & .729 & .15 & .618 & .17 \\
\hline 8 & .667 & .15 & .886 & .11 & .781 & .12 \\
\hline 9 & .888 & .12 & .847 & .19 & .867 & .17 \\
\hline 10 & .914 & .09 & .983 & .06 & .948 & .07 \\
\hline 11 & $.881^{*}$ & .08 & .936 & .06 & .908 & .05 \\
\hline 12 & .715 & .20 & .603 & .16 & .659 & .17 \\
\hline 13 & .628 & .09 & .850 & .14 & .739 & .12 \\
\hline 14 & .796 & .15 & .907 & .06 & .851 & .10 \\
\hline 15 & .743 & .13 & .854 & .07 & .798 & .10 \\
\hline 16 & .781 & .10 & .893 & .06 & .837 & .11 \\
\hline Mean & .724 & & .801 & & .762 & \\
\hline
\end{tabular}


$p<.001, M S \mathrm{e}=.01747$ ]; and the context $\times$ trial type interaction was also reliable $[F(15,165)=4.45$, $p<.001, M S e=.03202$ ] .

Despite the fact that each context pair differed only in the presence of the target line, the main effect of context indicated that there were reliable differences in the discriminability of the contexts. The lower overall accuracy rate for same trials was consistent with the noisy-operator model of perceptual matching. Finally, the reliable interaction indicated that the lower same-trial accuracy was not simply a bias for the subjects to respond "same" when uncertain. Instead, it suggested that it was easier to detect the sameness/difference of some contexts than that of others, as predicted by both the missing-feature and noisy-operator models.

Relations between subjective ratings and samedifferent accuracy. Table 4 shows simple and partial correlations between the ratings obtained in Experiment 2 and same-different accuracy in Experiment 3. The high level of agreement between the two types of correlations indicates that the rated stimulus properties were orthogonal to one another. As can be seen in Table 4, correlations were calculated for accuracy on the same trials alone (top panel), on the different trials alone (middle panel), and on the same and different trials combined (bottom panel).

The correlations for dimensionality showed that the ratings made under the free-viewing and 100-msec exposures were reliable predictors of accuracy on same trials, that none of the exposure conditions yielded reliable ratings on different trials, and that only the free-viewing condition yielded reliable ratings for the combined same and different trials.

In contrast to the dimensionality ratings, none of the dissimilarity ratings were positively related to accuracy. In fact, the free-viewing dissimilarity ratings were reliably negatively predictive of accuracy on same trials, and marginally negatively predictive on the combined same and different trials. The positive correlation between dimensionality ratings and same accuracy for the 16 contexts, and the negative correlation between dissimilarity

Table 4

Simple and Partial Correlations Between the Ratings in Experiment 2 and Same-Different Accuracy in Experiment 3

\begin{tabular}{|c|c|c|c|c|c|c|c|}
\hline \multirow[b]{2}{*}{ Accuracy } & \multirow[b]{2}{*}{ Correlation } & \multicolumn{3}{|c|}{ Dimensionality } & \multicolumn{3}{|c|}{ Dissimilarity } \\
\hline & & FV & 100 & 40 & FV & 100 & 40 \\
\hline Same & $\begin{array}{l}\text { Simple } \\
\text { Partial }\end{array}$ & $\begin{array}{l}.43 \\
.52 \dagger\end{array}$ & $\begin{array}{l}.62^{*} \\
.68^{*}\end{array}$ & $\begin{array}{r}.18 \\
-.04\end{array}$ & $\begin{array}{l}-.56 \dagger \\
-.63 \dagger\end{array}$ & $\begin{array}{l}-.20 \\
-.32\end{array}$ & $\begin{array}{l}.43 \\
.45\end{array}$ \\
\hline Different & $\begin{array}{l}\text { Simple } \\
\text { Partial }\end{array}$ & $\begin{array}{l}.22 \\
.24\end{array}$ & $\begin{array}{l}.13 \\
.14\end{array}$ & $\begin{array}{l}.16 \\
.20\end{array}$ & $\begin{array}{l}-.10 \\
-.13\end{array}$ & $\begin{array}{l}-.05 \\
-.08\end{array}$ & $\begin{array}{r}-.03 \\
.04\end{array}$ \\
\hline Combined & $\begin{array}{l}\text { Simple } \\
\text { Partial }\end{array}$ & $\begin{array}{l}.38 \\
.51 \dagger \\
\end{array}$ & $\begin{array}{l}.42 \\
.46\end{array}$ & $\begin{array}{l}.21 \\
.13\end{array}$ & $\begin{array}{l}-.37 \\
-.43\end{array}$ & $\begin{array}{l}-.14 \\
-.23\end{array}$ & $\begin{array}{l}.21 \\
.15 \\
\end{array}$ \\
\hline
\end{tabular}

Note-Partial correlations are between ratings of one stimulus property (e.g., dimensionality) and line-identification accuracy, with the ratings of the other stimulus property (e.g., dissimilarity) controlled. FV = free viewing, $100=100$-msec exposure, $40=40$-msec exposure. Reliable correlation coefficients: ${ }^{*} r(14)=.62, p<.01 . \quad \operatorname{tr}(14)=.50$, $p<.05$.

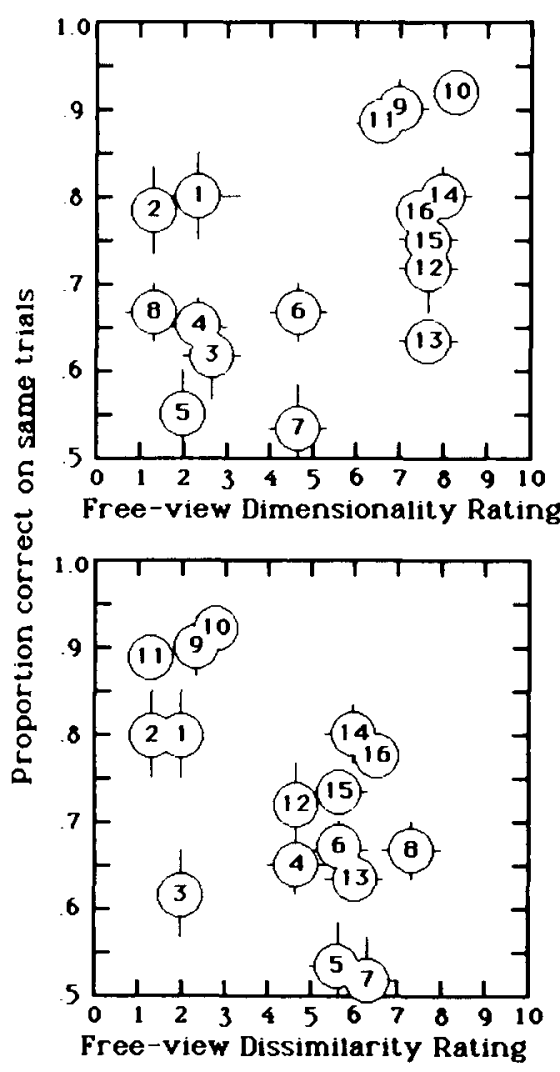

Figure 4. Top: Scattergraph of the relation between accuracy on same trials (Experiment 3) and free-viewing dimensionality ratings (Experiment 2). Bottom: Scattergraph of the relations between accuracy on same trials (Experiment 3) and free-viewing dissimilarity ratings (Experiment 2).

ratings and same accuracy, are shown as scattergraphs in Figure 4.

To ensure that the absence of reliable correlations between same-different accuracy and subjective ratings made under similar exposure conditions $(40 \mathrm{msec})$ was not an artifact of the smaller number of target presentations in the rating task, the same-different accuracy data was examined in only the first block of trials. The subjects received four views of each of the 16 contexts in the first block of the same-different task, and the identical number of each type of trial in the rating task as a whole. The accuracy results for only the first block of trials were relatively unchanged from the overall analysis presented previously. There were reliable differences in accuracy between the 16 contexts $[F(15,165)=5.90$, $p<.01]$, same trials did not vary reliably from different trials in accuracy $[F(1,11)=1.77]$, and the context $\times$ trial type interaction was reliable $[F(15,165)=3.70$, $p<.01]$. The correlation between accuracy in the first block of trials and overall accuracy for the 32 context $x$ trial type conditions was also reliable $[r(14)=.77$, $p<.011$.

Relations between line-identification and samedifferent accuracy. Line-identification accuracy in Ex- 
periment 1 ( $z$ scores in Table 1) was reliably correlated with same accuracy $[r(14)=.63, p<.01]$, with different accuracy $[r(14)=.52, p<.05]$, and with combined same and different accuracy $[r(14)=.69, p<.01]$. These three correlations did not differ reliably from one another $(p>.20)$.

Relative contributions of the three-dimensionality and perceptual discriminability to line-identification accuracy. Multiple regression analysis was used to examine the relative contributions of (1) the free-viewing dimensionality ratings from Experiment 2, and (2) the combined same-different accuracy from Experiment 3 to the line-identification accuracy in Experiment 1 ( $z$ scores in Table 1). The results indicated that these two variables combined were reliable predictors of lineidentification accuracy $(R=.90, p<.001)$, and that together they accounted for $81 \%$ of the total variation in line-identification accuracy. An examination of the partial correlations for each predictor (a correlation between one predictor and line-identification accuracy, with the effect of the other predictor held constant) indicated further that each predictor made a significant contribution to line-identification accuracy (dimensionality ratings, $p r$ $=.80, p<.01$; combined same-different accuracy, $p r$ $=.69, p<.01)$. A test of the interaction between the predictors did not account reliably for more variance than did the two predictors on their own $\left(R^{2}\right.$ change $=.02$, $p>.20$ ), indicating that each of the predictors made independent contributions to line-identification accuracy. The two partial correlations also did not differ reliably in magnitude from one another $(p>.20)$.

The multiple regression analysis was repeated for the dimensionality variable coded dichotomously (Contexts 1-8 vs. Contexts 9-16), for accuracy on same trials alone, and for accuracy on different trials alone. In each case, the same effects were reliable and of similar magnitude.

\section{Discussion}

The first finding of Experiment 3 was that same-different accuracy was predicted by free-viewing dimensionality ratings, just as line-identification accuracy was predicted by these ratings in Experiments 1 and 2. Thus, the three-dimensional contexts were perceptually more discriminable than the two-dimensional contexts in the present set of contexts. The implication of this finding is that it is important to control for discriminability differences when testing the claim that dimensionality has an effect on perceptual processing. Previous studies that did not control for discriminability are vulnerable to the criticism that this attribute was confounded with dimensionality (e.g., Earhard, 1987; Lanze et al., 1985).

The second finding was that false-different errors were reliably more prevalent than false-same errors, supporting a noisy-operator model of perceptual matching (Krueger, 1978). This finding is consistent with the view that emergent features act simply as additional sources of information concerning the target line (Enns \& Prinzmetal, 1984). It does not support the view that target lines are more perceptible in object-like contexts than in nonobject contexts because the higher order features of objects are processed more rapidly than their component parts (Pomerantz et al., 1977; Weisstein \& Harris, 1974).

The finding that dimensionality was correlated with discrimination accuracy primarily on the same trials meant that three-dimensional contexts led to fewer false-different errors than did two-dimensional contexts. According to the noisy-operator model, this suggests that internal noise is lower for representations of three-dimensional contexts than for those of two-dimensional contexts. In contrast, the finding that the correlation between dimensionality and discrimination accuracy was not reliable on different trials argues against the missing-feature model. This model would predict more false-same errors (lower different accuracy) for two-dimensional contexts than for threedimensional contexts because the two-dimensional contexts would be differentiated more slowly from their early blob-like representations.

Same-different accuracy was not predicted positively by dissimilarity ratings under any exposure conditions. In fact, the evidence indicated that dissimilarity ratings were negatively predictive of accuracy on same trials. This means that, in Experiment 2, more false-different errors were made on contexts rated as dissimilar than on contexts rated as similar. According to the noisy-operator model, this suggests that, under free-viewing conditions, internal noise is lower for representations of contexts rated as similar than for those of contexts rated as dissimilar. On different trials, the correlations were neither positively nor negatively predictive, providing no evidence for the role of false-same responses and, thus, no evidence for the missing-feature model. Even aside from the consideration of which model fits the discrimination data best, these findings show clearly that the dissimilarity ratings of the contexts were based on stimulus attributes different from those that influenced the perceptual discrimination of these contexts under tachistoscopic conditions.

The dissociation between dissimilarity ratings and discrimination accuracy points to the untenability of the assumption that context discriminability can be adequately assessed by dissimilarity ratings (Enns \& Prinzmetal, 1984; present Experiments 1 and 2). Previous hints that various form-discrimination measures do not agree with one another can be found in several places. For example, Beck (1982) and Julesz (1981) both warn that the dissimilarity of individual texture elements (forms) will predict texture segregation only when both levels of analysis (form and texture) involve the same preattentively discriminable differences (e.g., differences in line slope, length, or junctions). Differences related to transformations of form (e.g., line arrangement, reflection, or rotation) will be discriminable only at the level of the individual texture elements (form). Another example comes from work on alphanumeric recognition. Keren and Baggen (1981) have shown that the visual digits 6 and 9 are judged as more similar than the digits 6 and 8 in a similarity rating task, although the latter two are more confusa- 
ble in a digit-identification task. It is not possible to identify the stimulus properties used by the subjects in the present tasks, but the data show that ratings of dissimilarity were not influenced by the same factors that affected discrimination accuracy. Therefore, in future studies involving form discrimination, researchers should be very cautious about drawing inferences about perceptual discriminability from dissimilarity ratings.

One explanation for the discrepancy between dissimilarity ratings and tachistoscopic discriminability is that the relevant models of psychological similarity are different in the two tasks (Tversky, 1977). In particular, Gati and Tversky's $(1984,1987)$ distinction between conceptual and perceptual similarity may be relevant here. These authors have shown that for stimuli consisting of verbal descriptions and for tasks involving memory (conceptual), judgments of similarity/dissimilarity are influenced more by the features that the stimuli have in common than by their distinctive features. In contrast, the relative salience of common and distinctive features is reversed for pictorial stimuli and for tasks that emphasize encoding over memory (perceptual). Gati and Tversky $(1984,1987)$ predict that dissimilarity judgments will be based less on common features and more on distinctive features, as the task becomes more perceptual in nature.

To the extent that the free-viewing rating task in the present study permitted conceptual similarity to be assessed by the subjects, one would expect the relative salience of shared versus distinctive features to be weighted in favor of shared features. This prediction received some support in the pattern of correlations between the dissimilarity ratings and same-different accuracy (Table 4 and Figure 4). Contexts that were rated low in dissimilarity (i.e., had more shared features) tended to be those that also yielded high same accuracy in the discrimination task. Conversely, contexts that were rated high in dissimilarity (i.e., had fewer shared features) tended to be those that yielded low same accuracy. In other words, the ability of observers to make same judgments in the discrimination task predicted the dissimilarity ratings. The fact that there was no similar relation evident for accuracy on different trials suggests that the distinctive features were not nearly as important as the shared features in the rating task.

Evidence that distinctive features were weighted more heavily than shared features in the discrimination task came from the differences in accuracy already mentioned between same and different trials. The greater number of false-different errors relative to false-same errors suggests that observers were biased to focus on feature differences rather than on similarities in this task.

The third and most important finding of Experiment 3 was that same-different accuracy accounted for a sizable and reliable amount of variation in the line-identification data, independent of dimensionality. This supports Enns and Prinzmetal's (1984) claim that context discriminability affects the ability of subjects to identify a component line segment in a brief flash. In a simple linear model, same-different accuracy and dimensionality together accounted for $81 \%$ of the total variation in the OSE data. Furthermore, the contributions of these two factors were individually reliable, statistically independent of one another, and approximately similar in magnitude.

The fact that the correlation between same accuracy and line-identification accuracy $(r=.63)$ was somewhat larger than the correlation between different accuracy and lineidentification accuracy $(r=.52)$ is further evidence in support of Enns and Prinzmetal's (1984) view of the OSE. It suggests that line identification, like perceptual matching, depends on the amount of internal noise in the early representations. The reliable correlations between lineidentification accuracy and dimensionality further suggested that internal noise was generally lower in threedimensional than in two-dimensional contexts.

\section{GENERAL DISCUSSION}

This study demonstrated that three-dimensionality and discriminability contribute reliably, independently, and approximately equally to the accuracy with which subjects identify target lines embedded in noninformative line contexts (the object superiority and object-line effects). Although two previous studies had reported similar conclusions (Earhard, 1987; Lanze et al., 1985), the present study was the first to measure context discriminability independently from line-identification accuracy. Inadvertently, we also discovered that this was not a straightforward task.

Our original intent was to design a set of contexts in which dimensionality and discriminability were varied in an orthogonal manner. As a first approximation to these stimulus factors, we used subjective ratings provided by the subjects under free-viewing conditions. The mean ratings were consistent with our intuitions, yielding a stimulus space in which the two factors were orthogonally varied. However, ratings of dimensionality were found to be reliably related to line-identification accuracy, whereas ratings of dissimilarity were not found to be predictive of either line-identification or same-different accuracy. It was only when the dimensionality ratings were compared with data from the tachistoscopic same-different task that the contributions of threedimensionality and discriminability could be properly assessed.

The reliable effect of discriminability on lineidentification accuracy indicated that the subjects were using emergent features to help diagnose the identity of the target line. This conclusion is logically necessary because the contexts in which the lines were embedded were identical for each target alternative. The contexts, therefore, could not contribute to differences in discriminability on their own. The discriminability effect could have been mediated only by stimulus features that emerged from the interactions of target lines and contexts. As pointed out by Enns and Prinzmetal (1984), diagnostic emergent features are present at several levels of analysis in line draw- 
ings (e.g., global object identity and such local features as angles and bounded regions). Although the specific features that were relevant in any given context cannot be known without additional testing, their existence was clearly indicated by the effect of discriminability independent of differences in dimensionality.

Contrary to one of the implications of Enns and Prinzmetal's (1984) work, the independent effect of threedimensionality in the present study indicates that a complete account of the OSE cannot ignore this factor. The present dimensionality effect supports the claim made originally by Weisstein (Weisstein \& Harris, 1974; Williams \& Weisstein, 1978) that three-dimensionality alters the visual processing that a stimulus receives. However, the nature of the processing advantage for threedimensional contexts is still not clear. Weisstein's account suggests that three-dimensional contexts are processed more rapidly and accurately than two-dimensional or single-line contexts and, thus, that the target lines become available more rapidly to conscious perception in these contexts. Yet, this view was not supported by Enns and Prinzmetal (1984, Experiment 1), who found the OLE to be present only when there was a correlation between the emergent properties of contexts and the target lines. An account of the processing advantage for three-dimensional contexts needs to be developed that does not predict the increased perceptibility of individual target lines.

The independence of three-dimensionality and discriminability suggests that the dimensionality effect may reside in the contexts alone. That is, three-dimensional contexts (with or without target lines) may be processed more rapidly and accurately than two-dimensional contexts, whereas such factors as the presence of diagnostic emergent features may influence line-identification accuracy additively with this effect. This hypothesis could be tested by tachistoscopically presenting contexts without target lines to subjects and asking them to indicate whether a stimulus is present or absent. If the detection function over exposure duration grows more rapidly for threedimensional than for two-dimensional contexts (that are otherwise equated for discriminability when target lines are embedded), it would suggest that the visual system is indeed biased in favor of three-dimensional visual forms. Preliminary evidence supporting this hypothesis has recently been reported (Purcell, Stewart, \& Giacoletti, 1987).

Finally, it is important to bear in mind, in support of the main thrust of Enns and Prinzmetal's (1984) argument, that finding additive effects of dimensionality and discriminability on the OSE does not imply that these factors are equivalent in their explanatory status. That is, nothing in the present study negates the observation that three-dimensionality is neither a necessary nor a sufficient condition for the OLE. Indeed, the reliable OLE obtained for the two-dimensional contexts in the present study (Contexts 2, 5, 6, and 8 in Figure 1 and Table 1) reemphasizes the point that three-dimensionality is not a necessary condition. The lack of sufficiency for threedimensionality has also been shown repeatedly (e.g., Enns
\& Prinzmetal, 1984, Experiment 1; Klein, 1978; Lanze et al., 1985). On the other hand, differences in discriminability, coupled with a consistent mapping of the stimulus differences to target lines, have been shown to be necessary and sufficient to produce the OLE (Enns \& Prinzmetal, 1984). Three-dimensionality deserves only equivalent explanatory status if it can predict line-identification accuracy independently of discriminability differences between contexts and in the absence of target-line specification by emergent features.

\section{REFERENCES}

Ambler, B. FinkLEA, D. L. (1976). The influence of selective attention in peripheral and foveal vision. Perception \& Psychophysics, 19, 518-524.

Banks, W. P., Prinzmetal, W. (1976). Configurational effects in visual information processing. Perception \& Psychophysics, 19, 361-367.

BECK, J. (1972). Similarity grouping and peripheral discriminability under uncertainty. American Journal of Psychology, 85, 1-19.

BECK, J. (1982). Textural segmentation. In J. Beck (Ed.), Organization and representation in perception (pp. 285-317). Hillsdale, NJ: Erlbaum.

CHEN, L. (1982). Topological structure in visual perception. Science, 218, 699-700.

Chignell, M. H., Krueger, L. E. (1984). Further evidence for priming in perceptual matching: Temporal, not spatial, separation enhances the fast-same effect. Perception \& Psychophysics, 36, 257-265.

EARHARD, B. (1980). The line-in-object superiority effect in perception: It depends on where you fix your eyes and what is located at the point of fixation. Perception \& Psychophysics, 28, 9-18.

EARHARD, B. (1987). The object-line effect as an information-extraction strategy. Canadian Psychology, 28, 153. (Abstract)

EARHARD, B., ARMITAGE, R. (1980). From an object-superiority effect to an object-inferiority effect with movement of the fixation point. Perception \& Psychophysics, 28, 369-376.

EnNs, J. T., Prinzmetal, W. (1984). The role of redundancy in the object-line effect. Perception \& Psychophysics, 35, 22-32.

Eriksen, C. W., O'Hara, W. P., a Eriksen, B. A. (1982). Response competition effects in same-different judgments. Perception \& Psychophysics, 32, 261-270.

ESTES, W. K. (1972). Interactions of signal and background variables in visual processing. Perception \& Psychophysics, 12, 278-286.

Estes, W. K., \& TAYLOR, H. A. (1966). Visual detection in relation to display size and redundancy of critical elements. Perception \& Psychophysics, 1, 9-16.

Flom, M. C., Weymouth, F. W., \& Kahneman, D. (1963). Visual resolution and contour interaction. Journal of the Optical Sociery of America, 53, 1026-1032.

GATI, I., Trersky, A. (1984). Weighting common and distinctive features in perceptual and conceptual judgments. Cognitive Psychology, 16, 341-370.

GATI, I., TVERsky, A. (1987). Recall of common and distinctive features of verbal and pictorial stimuli. Memory \& Cognition, 15, 97-100.

GoTTSCHALDT, K. (1955). Gestalt factors and repetition. In W. D. Ellis (Ed. \& Trans.), A source book of Gestalt psychology. New York: Humanities Press. (Original work published 1926)

Helmholtz, H. (1962). Treatise on physiological optics (Vol. 3, J. P. C. Southall, Trans.). New York: Dover. (Original work published 1867)

Julesz, B. (1981). Textons, the elements of texture perception, and their interactions. Narure, 290, 91-97.

KEREN, G., BAGGEN, S. (1981). Recognition models of alphanumeric characters. Perception \& Psychophysics, 29, 234-246.

KLEIN, R. (1978). Visual detection of line segments: Two exceptions to the object superiority effect. Perception \& Psychophysics, 24, 237-242.

KLEIN, R. (1982). Patterns of perceived similarity cannot be general- 
ized from long to short exposure durations and vice versa. Perception \& Psychophysics, 32, 15-18.

Krueger, L. E. (1978). A theory of perceptual matching. Psychological Review, 85, 278-304.

Krueger, L. E., Chignell, M. H. (1985). Same-different judgments under high speed stress: Missing-feature principle predominates in early processing. Perception \& Psychophysics, 38, 188-193.

Lanze, M., Maguire, W., \& Weisstein, N. (1985). Emergent features: A new factor in the object-superiority effect? Perception \& Psychophysics, 38, 438-442.

Lanze, M., Weisstein, N., \& Harris, J. R. (1982). Perceived depth vs. structural relevance in the object-superiority effect. Perception \& Psychophysics, 31, 376-383.

LoCKHEAD, G. R. (1972). Processing dimensional stimuli: A note.Psychological Review, 79, 410-419.

MCClelland, J. L. (1978). Perception and masking of wholes and parts. Journal of Experimental Psychology: Human Perception \& Pefformance, 4, 210-223.

MCClelland, J. L., \&iller, J. (1979). Structural factors in figure perception. Perception \& Psychophysics, 26, 221-229.

Millspaugh, J. R. (1978). Effects of array organization on same-different judgments. Perception \& Psychophysics, 23, 27-35.

Navon, D. (1977). Forest before trees: The precedence of global features in visual perception. Cognitive Psychology, 9, 353-383.

PALMER, S. E. (1977). Hierarchical structure in perceptual representation. Cognitive Psychology, 9, 441-474.
Pomerantz, J. R., Sager, C. S., a Stoever, R. J. (1977). Perception of wholes and of their component parts: Some configural superiority effects. Joumal of Experimental Psychology: Human Perception \& Performance, 3, 422-435.

Prinzmetal, W. (1981). Principles of feature integration in visual perception. Perception \& Psychophysics, 30, 330-340.

Purcell, D. G., Stewart, A. L., Giacoletti, A. M. (1987, November). The object detection effect. Paper presented at the Annual Meeting of The Psychonomic Society, Seattle, Washington.

Treisman, A., Schmidt, H. (1982). Illusory conjunctions in the perception of objects. Cognitive Psychology, 14, 107-141.

Tversky, A. (1977). Features of similarity. Psychological Review, 84, 327-352.

Weisstein, N., Harris, C. S. (1974). Visual detection of line segments: An object-superiority effect. Science, 186, 752-755.

Weisstein, N., Wiluams, M. C., Harris, C. S. (1982). Depth, connectedness, and structural relevance in the object-superiority effect: Line segments are harder to see in flatter patuems. Perception, 11, 5-17.

Willams, A., Weisstein, N. (1978). Line segments are perceived better in coherent contexts than alone: An object-line effect. Memory \& Cognition, 6, 85-90.

Wolford, G. (1975). Perturbation model for letter identification. Psychological Review, 82, 184-199.

(Manuscript received May 5, 1987; revision accepted for publication March 25, 1988.) 